\title{
Inflammatory response to clozapine in the absence of myocarditis: case report
}

Patrick Davey, Siobhan Gee and Sukhi S. Shergill

\section{Summary}

A case is presented of a 25-year-old man with treatmentresistant paranoid schizophrenia whose only previous trial of clozapine had been stopped following a suspected clozapineinduced myocarditis. Due to the failure of his psychosis to respond to a number of antipsychotic treatments and augmentation strategies, clozapine was restarted on admission. His rechallenge was marked by intermittent pyrexia, tachycardia and elevated C-reactive protein (CRP), but eosinophilia was absent. Clozapine was started and then stopped twice following extensive investigation and with specialist cardiology consultation. Physical symptoms and CRP elevation resolved shortly after clozapine cessation. We believe this constituted an idiosyncratic systemic inflammatory response to clozapine treatment.

\section{Declaration of interest}

None.

\section{Copyright and usage}

(C) The Royal College of Psychiatrists 2016. This is an open access article distributed under the terms of the Creative Commons NonCommercial, No Derivatives (CC BY-NC-ND) licence.
Clozapine is the only antipsychotic drug to repeatedly demonstrate efficacy for patients suffering from schizophrenia that fails to respond to other medication. Unfortunately, clozapine is not only uniquely effective but also has a broad range of adverse effects. The rare side-effect of agranulocytosis is probably the best known, but clozapine has also been associated with myocarditis and cardiomyopathy. Myocarditis usually presents in the first 6-8 weeks after the start of treatment (median 3 weeks) ${ }^{1}$ but may occur at any time. The reported risk of fatal myocarditis varies widely, from 1 in 67000 in the USA $^{2}$ to a recent Australian study which estimated the risk of myocarditis at $1 \%$ of those treated (of whom 1 in 10 died). ${ }^{3}$ Given that the outcome may be fatal, contemporary practice is to monitor patients closely for signs and symptoms in the month of treatment. ${ }^{4}$ Patients who develop persistent tachycardia, fatigue, flu-like symptoms and unexplained fever should have a more comprehensive specialist assessment of their cardiac status. In confirmed clozapine-induced myocarditis, clozapine should be stopped. The characteristic clinical findings of myocarditis include electrocardiogram (ECG) abnormalities, increased creatine kinase (CK), increased troponin levels and eosinophilia.

In the first month of treatment, clozapine is known to increase levels of pro-inflammatory cytokines ${ }^{5}$ and to cause a subclinical rise in C-reactive protein (CRP), ${ }^{6}$ which may be linked to the development of fever and flu-like symptoms. These symptoms may be warning signs of damaging inflammatory processes in the internal organs (myocarditis, polyserositis), or may be apparently harmless and subsequently dissipate.

Stuhec ${ }^{7}$ describes the case of an 80 -year-old female patient with concurrent dementia who developed a raised CRP and fever on the 7th day of low-dose clozapine therapy. Her symptoms resolved on cessation of the clozapine, and rechallenge was not attempted. Similarly, Kohen et $a l^{8}$ report a 73 -year-old male, also with comorbid dementia, who developed a fever and raised CRP on day 15 of clozapine therapy which resolved on discontinuation but recurred on rechallenge. In both of these cases, neuroleptic malignant syndrome, agranulocytosis, infection and myocarditis were ruled out as possible underlying causes.
This report adds to existing evidence of idiosyncratic systemic inflammatory response to clozapine in the absence of myocarditis or polyserositis.

\section{Case}

\section{Presentation}

D.J. is a 25-year-old White British male with a diagnosis of treatment-resistant paranoid schizophrenia, complicated by ongoing poly-substance misuse. His first contact with psychiatric services was at the age of 15 when he attacked his father with a chisel during a psychotic episode. He was detained under the Mental Health Act and admitted to a psychiatric in-patient unit. Subsequently he has spent most of his adult life on psychiatric wards. On admission to our service he attributed many of his difficulties to an extensive history of multiple illicit substances including amphetamines, ecstasy, magic mushrooms, benzodiazepines and cannabis. He reported a desire to remain abstinent from drugs, and on admission and throughout the events below, urine drugs screens were negative. D.J. smoked throughout his admission, before, during and after Clozapine titrations. There was no medical history of note reported at admission when a complete set of routine blood tests were completed; this included an autoimmune screen, hepatitis and liver function tests which were all within normal ranges.

Prior to admission he had one previous trial of clozapine 8 years earlier. Two weeks after that initiation of clozapine he was admitted to a local general hospital with pyrexia, vomiting, headache and tachycardia. His ECG showed a sinus tachycardia with ectopics and $t$-wave abnormalities. An echocardiogram was completed which was normal. His CRP was elevated at $110 \mathrm{mg} / \mathrm{L}$ (reference range $<5$ ) and troponin I was raised $0.18 \mu \mathrm{g} / \mathrm{L}$ (reference range $<0.05$ ). A full blood count was normal. Following cessation of clozapine the above symptoms resolved spontaneously and the impression of the medical team was that this was a clozapineinduced myocarditis. Clozapine was therefore stopped.

Due to the unremitting nature of his psychosis and failure to respond to a number of antipsychotic treatments and augmentation strategies, the utilisation of clozapine was reconsidered on admission to our service. The potential benefits of clozapine were thought to outweigh the potential harm to D.J. Pre-clozapine $24 \mathrm{~h}$ 


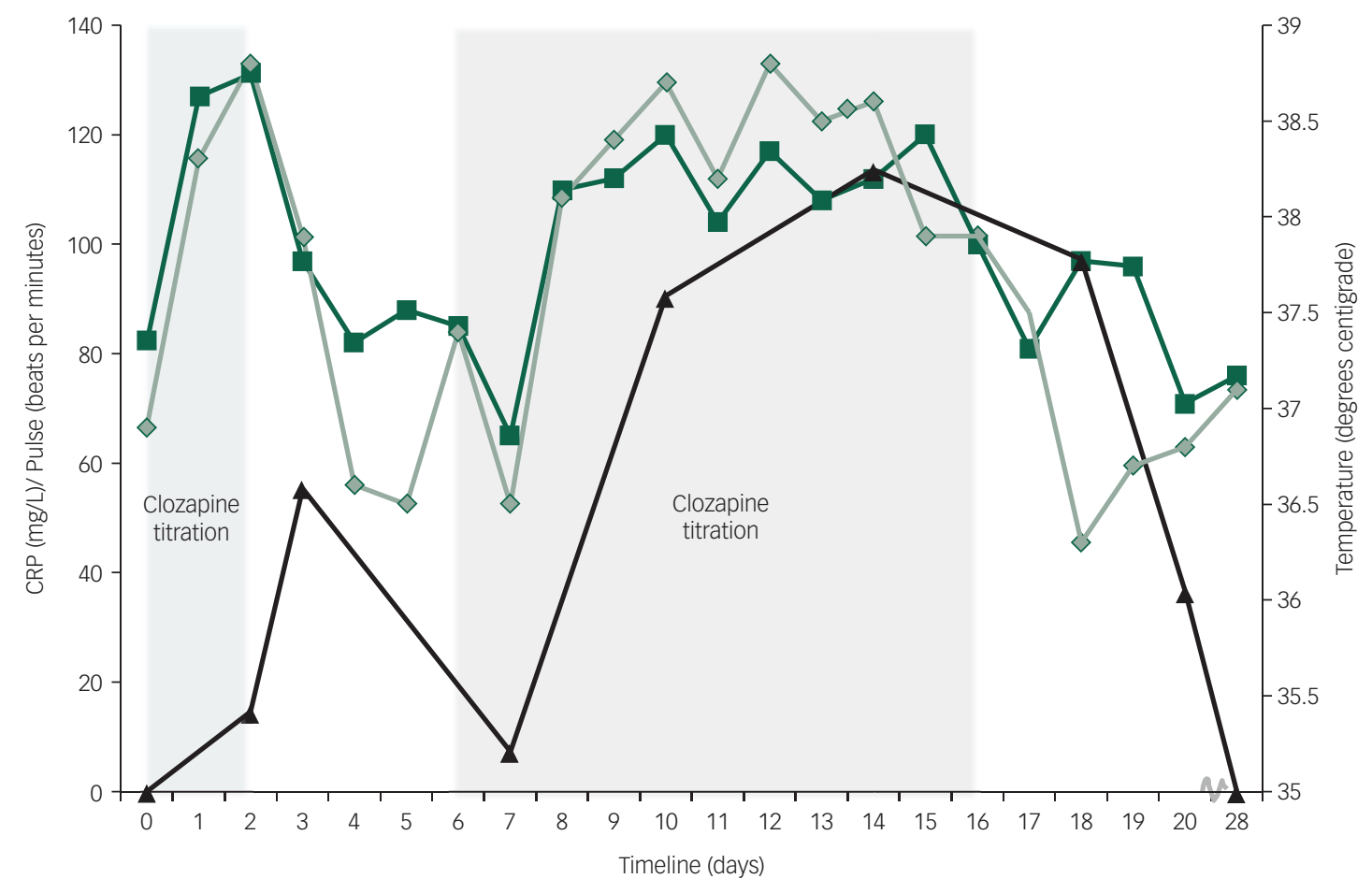

Pulse $\mathfrak{\imath}$ CRP $\hat{v}$ - Temperature

ambulatory cardiac monitoring and transthoracic echocardiograms revealed no abnormalities and an ejection fraction of $50-53 \%$. Specialist cardiology consultation supported rechallenge with clozapine, given the limited therapeutic options. Clinical observations were all within normal ranges as were baseline CRP $<5 \mathrm{mg} / \mathrm{L}$ and CK 99 IU/L (reference range $\leq 150$ ) measures. On admission his medication was olanzapine $15 \mathrm{mg}$ twice daily, amisulpride $1200 \mathrm{mg}$ once daily and omega-3 tablets. Amisulpride was slowly reduced and discontinued prior to starting clozapine and omeprazole was switched to lansoprazole $30 \mathrm{mg}$, once daily. Once the above was completed, treatment with clozapine was commenced under an offlicense agreement and olanzapine was reduced to $10 \mathrm{mg}$ once daily. Clozapine was titrated according to Maudsley Prescribing Guidelines recommendations.

Two days after beginning clozapine titration, D.J. had an episode of repeated vomiting with intermittent pyrexia $\left(38.3-38.8^{\circ} \mathrm{C}\right)$ and a tachycardia of $110-120$ beats/min. Clozapine was stopped. His neutrophil count remained normal $4.510^{9} / \mathrm{L}$ (reference range $=2.2-6.3$ ) with a normal white cell count $6.5210^{9} / \mathrm{L}$ (reference range $=4.00-11.00$ ) and normal eosinophil count $0.0110^{9} / \mathrm{L}$ (reference range $=0-0.4$ ). CRP was elevated at $55.4 \mathrm{mg} / \mathrm{L}$. There was no suggestion clinically or from an ECG of myocarditis or cardiomyopathy. After review, it was felt the episode was due to comorbid infection, possibly gastroenteritis, and in the absence of evidence of cardiac insult clozapine was re-commenced after D.J. was consulted. Although D.J. was treated under Section 3 of the MHA 1983, he was deemed to have capacity to be part of these decisions, and any decisions made were reached collaboratively with him and the involvement of his family.

Two days after the second clozapine rechallenge, D.J. began feeling unwell with diaphoresis, intermittent pyrexia and tachycardia. Clozapine was held, and the following investigations were completed. An ECG was repeated which showed a sinus tachycardia. A day later his CRP had risen to $90.6 \mathrm{mg} / \mathrm{L}$ while his
CK, eosinophil and neutrophil count remained within normal ranges. A septic screen found no infective source of the symptoms. His symptoms were considered to be an inflammatory process secondary to clozapine treatment. After consideration of the potential benefits and risks to D.J.'s physical and mental health, and after a detailed discussion with the patient and family, clozapine titration was continued.

The intermittent pyrexia continued and 4 days later his CRP had risen further to $113 \mathrm{mg} / \mathrm{L}$. At this stage, he was receiving clozapine $75 \mathrm{mg}$ twice daily. No clozapine plasma levels were taken as it was felt very unlikely that such a small dose could lead to toxicity. His neutrophil, white cell and eosinophilia counts remained normal. In view of the pyrexia and elevated CRP, a septic screen was repeated which found no source of infection. A measure of troponin I was negative $<0.05 \mu \mathrm{g} / \mathrm{L}$. Repeat echocardiography revealed no change in cardiac function. Cardiology consultation suggested that a normal troponin measure and echocardiogram did not support a diagnosis of myocarditis and a more likely scenario was of a systemic inflammatory response to clozapine driving a secondary tachycardia. In light of the significant and persistent physical symptoms of pyrexia, nausea, vomiting and tachycardia clozapine was stopped 2 days later. Mild abnormality in liver function with alkaline phosphatase 156 IU/L (reference range $=30$ 130) and Gamma GT 219 IU/L (reference range=1-55) was detected. Furthermore, a measure of amylase was modestly raised at $198 \mathrm{IU} / \mathrm{L}$ (reference range $\leq 100$ ), all of which supports the presence of a systemic inflammatory process (Fig. 1).

\section{Outcome and follow-up}

The impact of clozapine on his presentation was highlighted by the improvement in his physical health following cessation. There were no further episodes of pyrexia or tachycardia, and 4 days after clozapine was withdrawn his CRP had lowered (CRP $36 \mathrm{mg} / \mathrm{L}$ ). It was concluded that he had experienced an idiosyncratic systemic 
inflammatory response to clozapine, and further challenge with clozapine was contraindicated. Following the discontinuation of clozapine D.J.'s psychotic symptoms persisted and he showed little insight into his illness. Olanzapine was reinstated and he was later discharged to a rehabilitation setting on amisulpiride $300 \mathrm{mg}$ twice daily, olanzapine velotabs $30 \mathrm{mg}$ nocte, lansoprazole $30 \mathrm{mg}$ once daily, procyclidine $2.5 \mathrm{mg}$ once daily and omega- $31 \mathrm{~g}$ nocte.

\section{Discussion}

Maudsley Guidelines suggest clozapine initiation starts following satisfactory baseline CRP and troponin measures, echocardiography and normal physical observations. Once titration has started, in line with their recommendations, they propose careful monitoring for any signs or symptoms of developing myocarditis or cardiomyopathy especially in the first 3 months of treatment Symptoms of this would include flu-like symptoms, tachycardia, fever, dyspnoea and chest pain, while signs might include ECG changes (ST-depression), enlarged heart on radiology or echocardiography, eosinophilia, elevated CRP or troponin and evidence of heart failure. If a patient develops the above signs or symptoms, it is considered to be evidence of an adverse effect of clozapine on the myocardium and following a presumptive diagnosis of myocarditis clozapine is often discontinued.

This paper presents a similar clinical picture but in the absence of any direct impact on myocardium. This reinforces the need for specific investigations during clozapine titration to clarify specific cardiac changes to reduce presumptive diagnosis and ensure all is done to fully investigate a patient's symptoms and if necessary seek a cardiologist's advice.

This is an important observation because a presumptive diagnosis of clozapine-induced myocarditis is a contraindication to any subsequent rechallenge with clozapine. Although this patient was unable to continue with clozapine treatment, his diagnosis of what appears to have been a clozapine-induced idiosyncratic systemic inflammatory response was made following significant investigations. Our team was able to complete repeat echocardiography, CRP and troponin measures enabling us, with a cardiologist's advice, to rule out a clozapine-induced myocarditis. Given the dearth of therapeutic options in this patient group, diagnostic clarity becomes ever more salient to reduce unnecessary clozapine discontinuation, especially when considering the monitoring necessary during consideration of a clozapine rechallenge.

Improving diagnostic accuracy is developing, and within the Maudsley Guidelines they present a monitoring procedure developed in Australia ${ }^{9,10}$ - detecting $100 \%$ of symptomatic cases of myocarditis using serial measures of troponin, CRP and echocardiography. Furthermore, some authors argue that that Type B natriuretic peptide could be used to identify myocarditis. ${ }^{11}$ Despite some progress, we believe future directions for research should include increased validation of monitoring parameters to enable earlier and accurate diagnosis of myocarditis, so that decisions to stop clozapine use in patients are made as accurately as possible.

Patrick Davey, MBBS, PGDIP, Anxiety Disorders Residential Unit, Bethlem Royal Hospital, London, UK; Siobhan Gee, MPharm, MPhil, MRPharmS, MFRPSIl, PGDipGPP, Pharmacy Department, Bethlem Royal Hospital, London, UK; Sukhi S. Shergill, MBBS, SFHEA, PhD, FRCPsych, National Psychosis Unit, Bethlem Royal Hospital, London, UK

Correspondence: Patrick Davey, Anxiety Disorders Residential Unit, Bethlem Royal Hospital, London, UK. Email: patrick.davey@slam.nhs.uk

First received 15 May 2016, accepted 30 May 2016

\section{Funding}

S.S.S. acknowledges the support of the NIHR BRC and ERC Consolidator Award, National Psychosis Unit, Bethlem Royal Hospital.

\section{References}

1 La Grenade L, Graham D, Trontell A. Myocarditis and cardiomyopathy associated with clozapine use in the United States (Letter). N Engl J Med 2001; 345: 224-5.

2 Warner B, Alphs L, Schaedelin J, Koestler T. Clozapine and sudden death. Lancet 2000; 355: 842.

3 Haas SJ, Hill R, Krum H, Liew D, Tonkin A, Demos L, et al. Clozapine-associated myocarditis: a review of 116 cases of suspected myocarditis associated with the use of clozapine in Australia during 1993-2003. Drug Saf 2007; 30: 47-57.

4 Taylor D, Paton C, Kapur S. Maudsley Prescribing Guidelines, 11th edn. Informa Healthcare, 2012.

5 Roge R, Moller BK, Andersen CR, Correll CU, Nielsen J. Immunomodulatory effects of clozapine and their clinical implications: what have we learned so far? Schizophr Res 2012; 140: 204-13.

6 Loffler S, Loffler-Ensgraber M, Fehsel K, Klimke A. Clozapine therapy raises serum concentrations of high sensitive C-reactive protein in schizophrenic patients. Int Clin Psychopharmacol 2010; 25: 101-6.

7 Stuhec M. Clozapine-induced elevated C-reactive protein and fever mimic infection. Gen Hosp Psychiatry 2013; 35: 680.

8 Kohen I, Afzal N, Hussain S, Manu P. Increases in C-reactive protein may predict recurrence of clozapine-induced fever. Ann Pharmacother 2009; 43: 143-6.

9 Ronaldson KJ, Fitzgerald PB, Taylor AJ, Topliss DJ, MCNeil JJ. Clozapine-induced myocarditis and baseline echocardiography. Aust N Z J Psychiatry 2012; 46 : 1006-7.

10 Ronaldson KJ, Fitzgerald PB, Taylor AJ, Topliss DJ, McNeil JJ. A new monitoring protocol for clozapine-induced myocarditis based on an analysis of 75 cases and 94 controls. Aust N Z J Psychiatry 2011; 45: 459-65.

11 Layland J, Liew D, Prior D. Clozapine-induced cardiotoxicity: a clinical update. Med J Aust 2009; 190: 190-2. 